\title{
$\alpha 2,3-$ and $\alpha 2,6-$ linked sialic acids are important for cell binding and replication of Newcastle disease virus in chicken primary neuronal cells
}

\author{
Y. GUO, J. ZHAO, X. CHANG, W. YAO, H. WANG, W. WANG, X. WANG, S. ZHANG, Z. YANG, S. XIAO*
}

College of Veterinary Medicine, Northwest a \& F University, Yangling 712100, Shaanxi, P. R. China

Received January 29, 2018; accepted April 19, 2018

\begin{abstract}
Summary. - Velogenic Newcastle disease virus (NDV) causes encephalitis and severe neurological disorders in avian species. Sialic acid (SA) has been considered as a receptor for NDV infection and determining tissue tropism. The neurotropic mechanism of NDV in birds is completely unknown. Here we have investigated the role of viral receptor SA in neurotropism of NDV in chickens. We determined that $\alpha 2,3$ - and $\alpha 2,6$-linked SA receptors were implicated in NDV encephalitis and viral binding to primary neuronal cells using immunohistofluorescence and virus-cell binding assay. Both SA receptors were found to co-localize with velogenic strain F48E9 in neuropathological lesions of chicken brains after infection through intraocular-nasal routes. The replication of velogenic F48E9 in primary neuronal cells was more efficient than that of lentogenic strain LaSota. The virus-neuronal cell binding capabilities of both the velogenic and the lentogenic strains have no difference. Furthermore, the cell-binding capability and the replication of both strains were significantly decreased by pretreatment with sialidases in neuronal cells. These results demonstrated that $\alpha 2,3-$ and $\alpha 2,6$-linked SA receptors were important for the initiation of NDV infection in the chicken nervous system. This study should provide preliminary evidence for a better understanding of the neurotropism of NDV in chickens.
\end{abstract}

Keywords: Newcastle disease virus; sialic acid; chicken; primary neuronal cells

\section{Introduction}

Newcastle disease is a highly contagious avian disease that causes huge economic losses in the poultry industry (Alexander, 1997). Its causative agent is Newcastle disease virus (NDV), an enveloped, non-segmented, single-stranded, negative sense RNA virus that belongs to the genus Avulavirus within the family Paramyxoviridae (Lamb et al., 2007). Based on its different pathogenesis and virulence in chickens, NDV has been divided into three pathotypes: lentogenic (low

*Corresponding author. E-mai: saxiao@nwafu.edu.cn; phone: +86-029-87091117.

Abbreviations: $\mathrm{EID}_{50}=50 \%$ egg infective dose; $\mathrm{hpi}=$ hours post infection; dpi = days post infection; $\mathrm{MAA}=$ Maackiaa murensis lectin; $\mathrm{NDV}=$ Newcastle disease virus; $\mathrm{NeuA}=\alpha 2-3,6,8,9$ neuraminidase A; NP = nucleoprotein; $\mathrm{SA}=$ sialic acid; $\mathrm{SNA}=$ Sambucus nigra agglutinin; $\mathrm{SPF}=$ specific pathogen-free; $\mathrm{TCID}_{50}=50 \%$ tissue culture infective dose; VCNA = Vibrio cholerae neuraminidase; VNND = velogenic neurotropic Newcastle disease; VVND = velogenic viscerotropic Newcastle disease virulence), mesogenic (medium virulence) and velogenic (high virulence) (Alexander, 2000). The lentogenic virus causes minimal infections or subclinical to mild respiratory infections in younger animals (Alexander and Senne, 2008). The mesogenic virus in general is associated with a low mortality rate and causes mild respiratory or occasional nervous system symptoms. The velogenic viruses can lead to high morbidity and mortality and has been further divided into velogenic viscerotropic Newcastle disease (VVND) and velogenic neurotropic Newcastle disease (VNND) based on their behaviour in adult chickens after cloacal inoculation. The VVND strain causes hemorrhagic intestinal lesions and high mortality. VNND causes mainly nervous system symptoms and less severe or no visceral lesions in chickens and lower mortality, when compared with VVND (Brown et al., 1999; Cattoli et al., 2011). Importantly, virulent strains, including VNND and sometimes VVND, can cause encephalitis and neurological impairments (Ecco et al., 2011). The clinical symptoms caused by the VNND virus lead to significant neurological disorders, such as twisted neck, head and neck 
tremors, circling, paralysis of legs or wings, opisthotonus, paralysis/paresis and other symptoms; the mortality rate in chickens can reach $90 \%$ or higher (Moura et al., 2016).

Sialic acid (SA), a monosaccharide with a nine-carbon backbone, namely $\mathrm{N}$-acetylneuraminic acid (Neu5Ac), is frequently found at the terminal ends of gangliosides (glycosphingolipids) and bound to galactose (Gal) in N- and $\mathrm{O}$-glycoproteins of animal tissues. SA serves as a receptor of NDV (Ferreira et al., 2004). The specific linkage of SA residues to glycoconjugates is believed to be one of the major determinants of viral tropism for paramyxoviruses (Villar and Barroso, 2006), orthomyxoviruses (Viswanathan et al., 2010), polyomaviruses (Dugan et al., 2008; Neu et al., 2009), rotaviruses (Delorme et al., 2001), coronaviruses (Schultze et al.,1992) and adenoviruses (Dechecchi et al., 2001). The specificity of influenza virus strains is related to the SA linkage $(\alpha 2,3$ or $\alpha 2,6)$ and the type of SA residue (NeuAc, NeuGc or 9-O-Ac-NeuAc) (Schnaar, 1991). It is widely believed that human influenza virus mainly recognizes a2,6-linked SA and that avian influenza viruses prefer $\alpha 2,3$-linked SA (Stevens et al., 2006; Suzuki, 2005), whereas NDV binds to gangliosides with both terminal $\alpha 2,3$ - and $\alpha 2,6$-linkages (Ferreira et al., 2004). Both $\alpha 2,3$ - and $\alpha 2,6$-linked SA receptors are simultaneously distributed in the susceptible tissues of chickens (Wang et al., 2012). Sialidase and lectin treatments also revealed that NDV uses both a2,3- and a2,6- linked sialic acids in ELL-0 cells (Sánchez-Felipe et al., 2012). In addition, the receptor specificity of NDV seems to be more complex than the binary model of SA $\alpha 2,3$ and SA $\alpha 2,6$ binding. To infect host cells, this virus may use a wide range of SA moieties, or even other pathways (Sánchez-Felipe et al., 2012).

Although NDV requires sialylated glycoconjugates for viral binding to host cells, the role of the specific linkage of SA in viral neuroinfection is still unknown. In this study, we provided preliminary evidence for the $\alpha 2,3$ and $\alpha 2,6$ SA linkage requirements for the specificity of NDV neuroinfection. Our results suggest that NDV uses $\alpha 2,3$ - and $\alpha 2,6$-linked SA to bind to the neuronal cells, and both SA receptors play a critical role in viral neural cell-binding and replication in the chicken nervous system.

\section{Materials and Methods}

NDV infection in chickens. The strain LaSota is a lentogenic vaccine virus (Yebio, China). The velogenic strain F48E9 is widely used as a standard challenge virus in China. The viruses were inoculated into 9- to 11-day-old specific pathogen-free (SPF) embryonated chicken eggs. Allantoic fluids containing viruses were collected and stored at $4^{\circ} \mathrm{C}$. Four-week-old SPF white Leghorn chickens (SPAFAS Poultry, China) in isolators were provided with food and water ad libitum. All experimental procedures were approved by the Animal care and use committee of the Northwest A \& F University, P. R.
China. The experiments were conducted in accordance with the guidelines of the Chinese committee for animal experiments in chickens (Protocol No. 2015ZX0512008-012).

Twenty-seven chickens were equally divided into three groups. Group 1 and group 2 were inoculated through intraocular-nasal routes with $0.2 \mathrm{ml}$ per bird of PBS containing $10^{6} \mathrm{EID}_{50}$ of F48E9 and LaSota viruses, respectively. Group 3 was inoculated with PBS as a control. The chickens were monitored daily for clinical signs and euthanized when they displayed severe neurological dysfunction symptoms and were moribund. Each brain sample was divided into two parts: one part was immediately frozen in liquid nitrogen for RNA isolation, while the other part was fixed with $4 \%$ paraformaldehyde for histopathological analysis.

Quantitative RT-PCR. Total RNA was extracted from infected chicken brain tissues and primary neuronal cells using the RNAiso plus reagent according to the manufacturer's instructions (Vazyme Biotech, China). The cDNA synthesis was carried out using the EasyScript ${ }^{\oplus}$ first-strand cDNA synthesis super mix (TransGene Biotech, China). The PCR was carried out on a Bio-Rad iQ5 real time PCR detection system using an EvaGreen Qpcr master mix (Abm, Canada). A standard curve was established with serially diluted plasmids $\left(10^{2}-10^{8}\right.$ copies $\left./ \mu \mathrm{l}\right)$ that harboured the $\mathrm{P}$ gene of NDV. The PCR product size was $322 \mathrm{bp}$ long and the primers were reported previously (Wang et al., 2017). SYBR Green qPCR detection system (Vazyme Biotech) and a real-time thermocycler (Four-channel, Tianlong, China) were used for qPCR analysis according to the manufacturer's instructions. Absolute quantification of viral copies was determined by comparison with the standard curve. A melting curve analysis was used to evaluate the amplification results.

Preparation of polyclonal antiserum against the nucleoprotein (NP) of NDV. The LaSota virus in allantoic fluids was purified on a sucrose gradient, and the virion proteins were separated on a $10 \%$ SDS-polyacrylamide gel. The gel was washed with distilled water and stained with $0.25 \mathrm{~mol} / \mathrm{l} \mathrm{KCl}$ for $20 \mathrm{~min}$. The NP-specific band was viewed in a black background and excised from the gel, followed by destaining with distilled water until the band disappeared. The excised gel band was minced and mixed with elution buffer (50 mmol/l Tris-HCl pH 8.0, 1 mmol/l EDTA, 1 mmol/l DTT, 150 $\mathrm{mmol} / \mathrm{l} \mathrm{NaCl}$ and $0.1 \%$ SDS in $1000 \mathrm{ml}$ ) and boiled for $10 \mathrm{~min}$. After one centrifugation, the eluted protein in the supernatant was quantified, and $0.5 \mathrm{mg}$ of protein was mixed with complete Freund's adjuvant (Sigma-Aldrich, USA) and injected subcutaneously into two 10-week-old guinea pigs. Booster immunizations were given twice with $0.5 \mathrm{mg}$ of protein mixed within complete Freund's adjuvant at 14-day intervals. The antisera were collected

Table 1. Primers used in qRT-PCR

\begin{tabular}{llll}
\hline Gene & Primer & Sequence & Acc. No. \\
\hline NDV & Forward & 5'-CAATAAATCGTCCAATGCTAA-3' & KP027403 \\
& Reverse & 5'-CTCCATCATAGACATCATCGA-3' & \\
\hline
\end{tabular}


for 2 weeks after the third immunization. The antiserum was tested by Western blot and ELISA.

Histopathology. The 4\% paraformaldehyde-fixed brain tissues were dehydrated, embedded in paraffin wax and sectioned from the mid-sagittal plane at a $4-\mu \mathrm{m}$ thickness. The sections from mockinfected birds were used as controls. The tissues were deparaffinized, rehydrated and subsequently stained with hematoxylin-eosin (HE) to detect histopathological changes under the microscope (Nikon, Japan). Each anatomical region of the brain was observed according to the partition of the chicken brain as previously described (Reiner et al., 2004; Ecco et al., 2011).

Immunohistochemistry and immunohistofluorescence. The chicken brain tissues were examined for SA by employing the lectindigoxigen in (DIG)-labeled Maackiaa murensis lectin (MAA), specific for alpha-2,3-linked SA, and the lectin-DIG-labeled Sambucus nigra agglutinin (SNA), specific for alpha-2,6-linked SA (Roche, Germany). Lectin histochemistry was performed as previously described (Cerna et al., 2002). Briefly, paraffin-embedded tissue sections were deparaffinized and immersed in $3 \%$ hydrogen peroxide to eliminate the endogenous peroxidase activity. The sections were treated with $5 \%$ chicken normal serum to avoid nonspecific staining and then incubated with DIG-labelled MAA $(5 \mu \mathrm{g} / \mathrm{ml})$ or SNA $(1 \mu \mathrm{g} / \mathrm{ml})$ at $4^{\circ} \mathrm{C}$ overnight. The sections of same tissues were incubated with PBS instead of the lectins as negative controls. After washing two times with $\mathrm{PBS}$, the sections were incubated with AP-labeled anti-DIG Fab fragments for $1 \mathrm{~h}$ at $37^{\circ} \mathrm{C}$. Bound lectin was visualized using the NBT/BCIP substrate. Immunohistofluorescence was performed by using anti-Digoxin-FITC $(20 \mathrm{mg} / \mathrm{ml}$, Sigma-Aldrich). The sections were incubated with anti-NDV NP antiserum (1:1000) overnight at $4^{\circ} \mathrm{C}$ after blocking with $5 \%$ bovine serum albumin (BSA) for $30 \mathrm{~min}$, followed by Alexa Fluor ${ }^{\circledR}$ 594conjugated AffiniPure donkey anti-guinea pig $\operatorname{IgG}(\mathrm{H}+\mathrm{L})$ (Jackson ImmunoResearch Lab, USA) for $30 \mathrm{~min}$ at $37^{\circ} \mathrm{C}$. Finally, the samples were counterstained with DAPI (Vector, USA).

Preparation of chicken primary neuronal cells. The brains were removed from 9-day-old chicken embryos and washed with sterile PBS. They were cut into pieces and trypsinized with $0.25 \%$ trypsin (Sigma-Aldrich) at $37^{\circ} \mathrm{C}$ for $5 \mathrm{~min}$. After trypsinization, the cells were resuspended in $10 \mathrm{ml}$ of DMEM/F12 (1:1) culture medium supplemented with $10 \%$ FCS and $1 \%$ antibiotic mix $(100 \mathrm{U} / \mathrm{ml}$ penicillin and $100 \mu \mathrm{g} / \mathrm{ml}$ streptomycin (Gibco). The suspension was sequentially filtered through $149 \mu \mathrm{m}, 74 \mu \mathrm{m}$ and $47 \mu \mathrm{m}$ cell strainers. The cells were counted and then cultured at approximately $10^{6}$ cells per well in a poly-L-lysine coated 12-well plate. After the cell attachment, the medium was removed, and the cells were washed three times with PBS. Neurobasal medium supplemented with $2 \%$ B-27 serum-free supplement (Invitrogen, USA) and L-glutamine (Solarbio, China) was then added. The cells were incubated at $37^{\circ} \mathrm{C}$ in a $5 \% \mathrm{CO}_{2}$ incubator.

Virus titration. The infected chicken brain tissues were homogenized. The supernatants were collected after centrifugation. The cultured media of the infected chicken primary neuronal cells were harvested at 12, 24, 36, 48, 60 and $72 \mathrm{~h}$ post infection (hpi). Virus tit- ers were determined by a $50 \%$ tissue culture-infective dose $\left(\mathrm{TCID}_{50}\right)$ in DF-1 cells with an anti-NP polyclonal antibody and a 50\% egg infectious dose $\left(\mathrm{EID}_{50}\right)$ by the method of Reed and Muench.

Confocal immunofluorescence assay. Chicken primary neuronal cells were infected with F48E9 at MOI of 0.01 and with LaSota at MOI of 1 . Briefly, the cells were fixed and permeabilized with $10 \%$ neutralized buffered formalin, followed by staining with MAA and SNA for lectins, an anti-NP polyclonal antibody or an anti-neuron specific beta-III tubulin antibody (Abcam). The cells were also counterstained with DAPI for detection of the nuclei. The cells were stained with secondary antibodies Alexa Fluor ${ }^{\circledR}$ 594-conjugated AffiniPure donkey anti-guinea pig IgG $(\mathrm{H}+\mathrm{L})$ and DyLight 488 rabbit anti-rat IgG (Jackson ImmunoResearch Lab, USA). The stained cells were observed by confocal microscopy (Nikon).

Virus-cell binding assay. The chicken primary neuronal cells in the 12-well plate were cooled to $4^{\circ} \mathrm{C}$ for $10 \mathrm{~min}$ and inoculated with strain F48E9 or LaSota at MOI of 50 at $4^{\circ} \mathrm{C}$ for 5, 15, 30, 60 and 90 $\mathrm{min}$. The cells were washed with cold PBS to remove unbound virus and harvested by scraping into the culture medium. The cell-bound viruses were determined by the $\mathrm{EID}_{50}$ method.

Neuraminidase treatment. Enzymatic removal of cell surface SA residues was achieved by pretreatment with Vibrio cholerae neuraminidase (VCNA) (Sigma-Aldrich), which can cleave both $\mathrm{N}$ - and $\mathrm{O}$-sialic acids from glycolipids and glycoproteins, and $\mathrm{\alpha} 2-3$, $6,8,9$ neuraminidase A (NeuA) (NEB, USA), which has a preference for cleaving $\alpha 2-3,6,8,9 \mathrm{~N}$-sialic acids. The SA $\alpha 2,6-\mathrm{Gal}$ or SA $\alpha 2,3-$ Gal of chicken primary neuronal cells was enzymatically eliminated by NeuA and VCNA as described previously (Wu et al., 2014). Briefly, the chicken primary neuronal cells in 12 -well plates were washed with OptiMEM and treated with $0.05 \mathrm{U} / \mathrm{ml}$ VCNA and $0.128 \mathrm{U} / \mathrm{ml}$ of NeuA at $37^{\circ} \mathrm{C}$ for $1 \mathrm{~h}$. After the sialidases were removed by washing with $\mathrm{PBS}$, the following 2 experiments were performed. In the first experiment, the sialidase-treated cells were infected with strains F48E9 or LaSota at MOI of 50 at $4^{\circ} \mathrm{C}$ for $2 \mathrm{~h}$. After washing three times with PBS, the cells were harvested with scraper. In the second experiment, the cells were infected with strains F48E9 or LaSota at MOI of 1 at $4^{\circ} \mathrm{C}$ for $1 \mathrm{~h}$. After washing three times with PBS, the cells were incubated with new medium with neuraminidase at $37^{\circ} \mathrm{C}$ to avoid live cells synthesizing new SA and shuttling new glycoforms to the plasma membrane, and neuraminidase was added every $12 \mathrm{~h}$ to prevent its degradation. The supernatants of the infected cells were collected at $6,12,24,48$ and $72 \mathrm{hpi}$. The virus was titrated by the $\mathrm{EID}_{50}$ method.

Statistical analysis. A comparison of virus titration data on the NDV RNA load in chicken samples was analyzed for statistical significance by the Mann-Whitney u test (paired Student's $t$-test). The data were presented as the mean \pm SD derived from at least three separate and independent experiments, with a value of $\mathrm{P}<0.05$ considered to be statistically significant. 
(a)

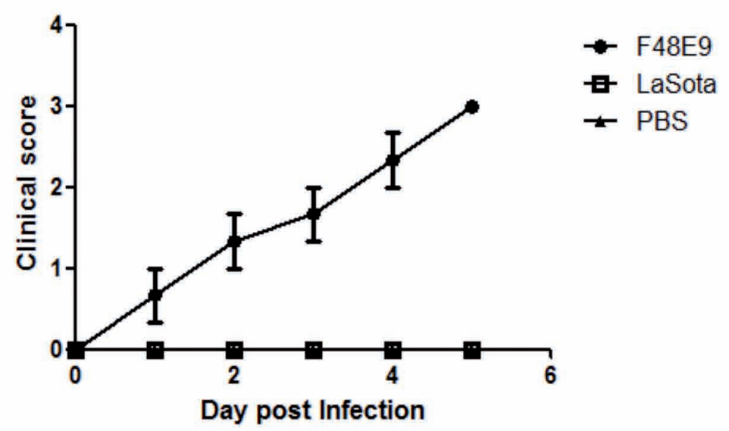

(c)

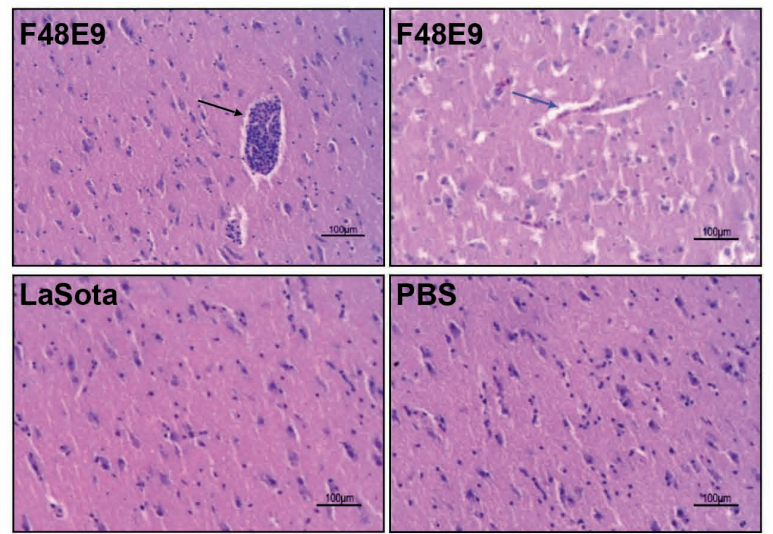

(b)

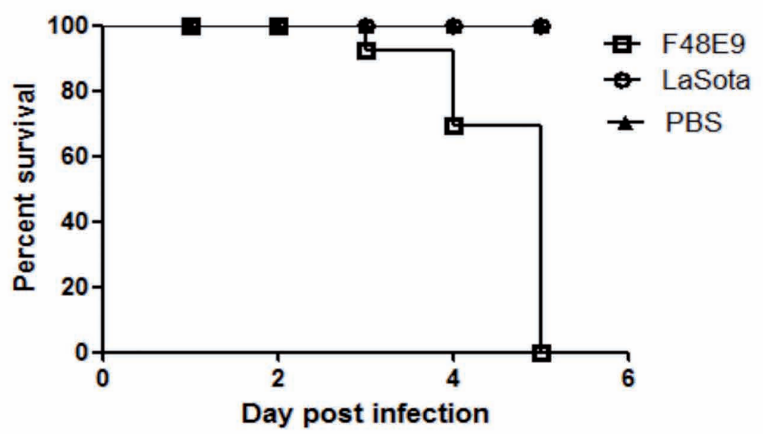

(d)

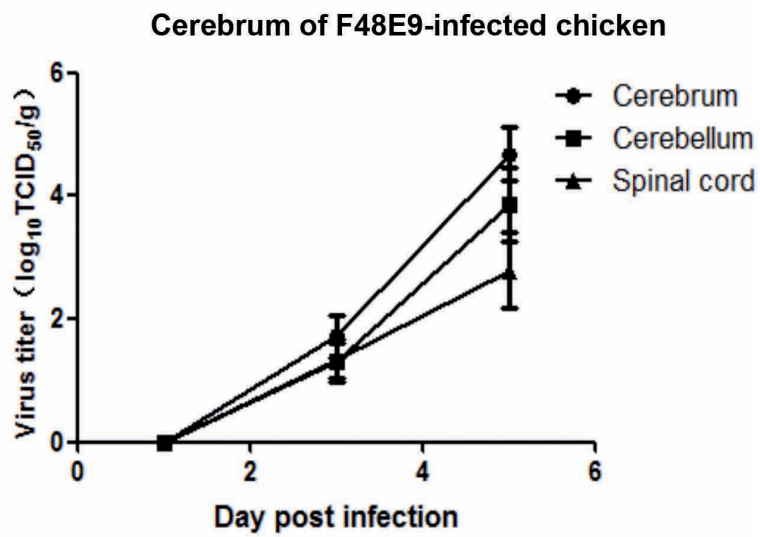

Fig. 1

Clinical diseases and viral replication in NDV-infected chickens

Four-week-old SPF chickens were inoculated through intraocular-nasal routes with $10^{6} \mathrm{EID}_{50} /$ bird of velogenic F48E9 and lentogenic LaSota viruses and then observed daily for clinical signs and mortality. (a) Clinical signs were scored as follows: 0 , healthy; 1 , slight illness (wings slightly drooping, cough, slightly depressed, slight crop effusion); 2, severe illness (severe depression, neck twisting, eyes fully closed, no response to external stimuli, decreased appetite, extreme difficulty breathing, crop exuding liquid); 3, dead. (b) Survival proportions of the NDV-infected chickens. (c) Neuropathological lesions of encephalitis caused by the F48E9 virus. The chicken brains at 5 days post-infection (dpi) were analyzed by HE staining. The infiltration of lymphocytes and plasma cells were observed around the vascular wall (black arrow) and vacuoles (blue arrow). Scale bars represent $100 \mu \mathrm{m}$. (d) Growth kinetics of the F49E9 virus in the chicken CNS. The viral titers were determined by a TCID ${ }_{50}$ assay. Values represent the averages of the results from three independent experiments.

\section{Results}

\section{Clinical signs}

Four-week-old SPF chickens infected through natural routes with velogenic strain F48E9 showed mild depression, head twitching and slightly dropping wings at 2 days post infection (dpi). The birds displayed coughing, dyspnea, loss of appetite, diarrhea, slight crop effusion, lateral recumbency and paralysis at $3 \mathrm{dpi}$. Severe neurological signs, such as neck twisting, unilateral leg paralysis accompanied with no response to external stimuli, obvious dyspnea, asthma, decreased appetite and excretion of crop liquid and green thin excrement, were additionally found at 4 dpi (Fig. 1a). All the F48E9 infected birds died between 3 and 5 dpi (Fig. 1b). No apparent clinical signs were observed in the LaSota and
PBS-control inoculated birds during the experimental days. Clinical signs were scored as follows: 0 , healthy; 1 , slight illness (wings slightly drooping, cough, slightly depressed, slight crop effusion); 2, severe illness (severe depression, neck twisting, eyes fully closed, no response to external stimuli, decreased appetite, extreme difficulty breathing, crop exuding liquid); 3 , dead. These results indicated that the velogenic F48E9 virus, as a VNND virus, causes typical neurological disorders.

\section{Encephalitis caused by NDV in chickens}

The neurologic lesions in the chicken brains infected with strains F48E9 and LaSota at 5dpi were examined by histopathological analysis. The lesions in the F48E9infected brain tissues showed perivascular cuffing and 
(a)

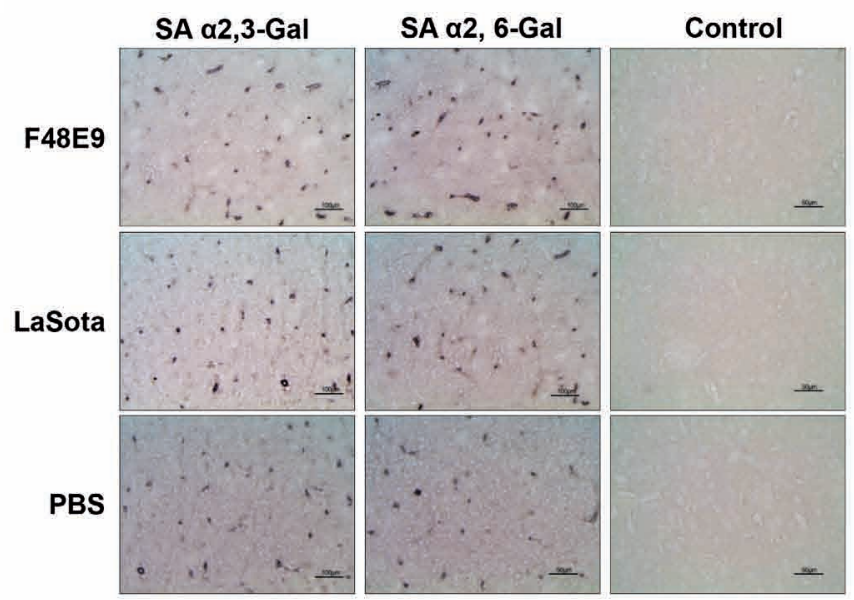

(b)

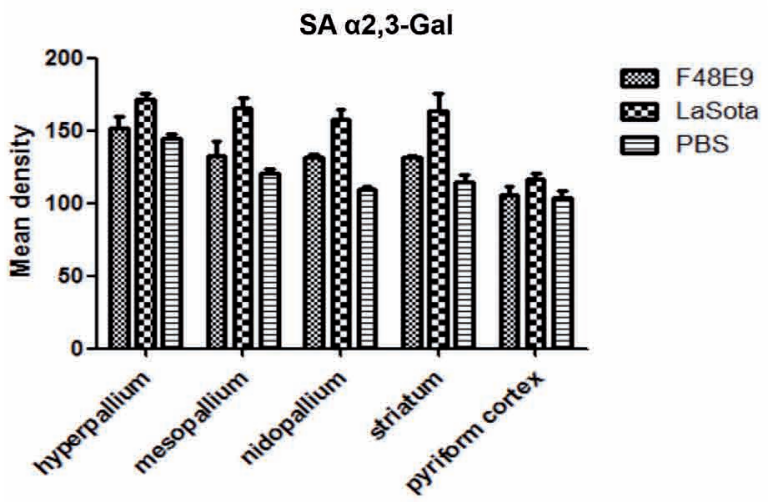

(c)

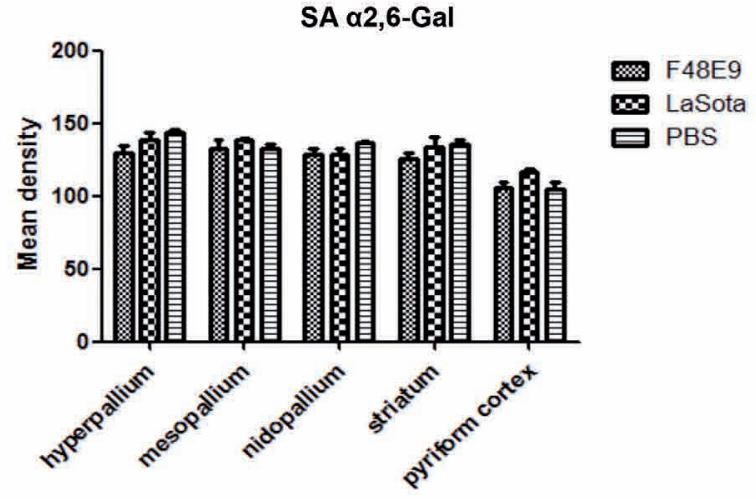

(d)

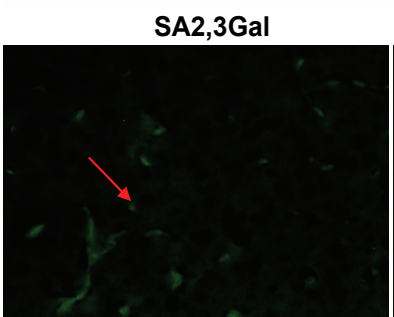

NP

DAPI

Merge
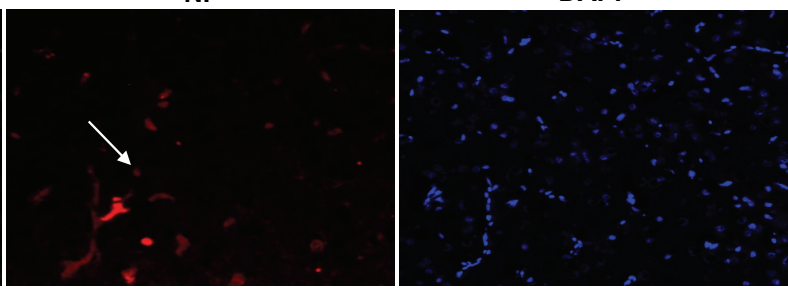

DAPI

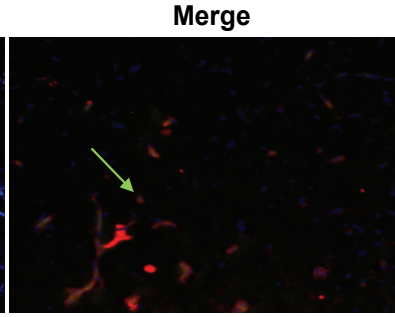

NP

Merge
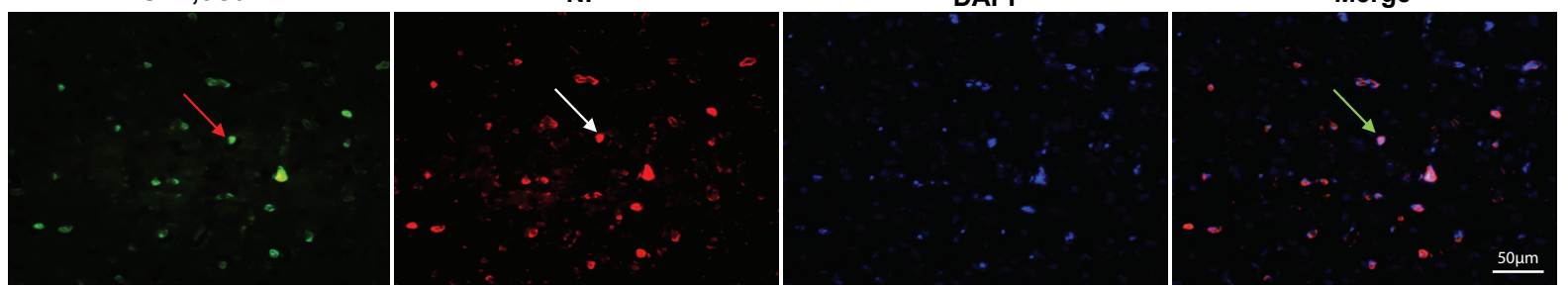

Fig. 2

Determination of SA receptors and NDV in the infected chicken brains

(a) SA receptors detected by immunohistochemistry analysis. The SA-positive coarse granules (blue-violet color) were found in most regions of the brain. Tissues incubated without a primary antibody treatment were used as negative controls. Hyperpallium showed a region with serious hemorrhage and hyperaemia. Scale bars represent $100 \mu \mathrm{m}$. (b) and (c) show the mean densities of stained lectin-SA $\alpha 2,3-\mathrm{Gal}$ and SA $\alpha 2,6$-Gal in various regions of the brains, respectively. The data were analyzed by Image-Pro Plus. (d) Co-localization of SA receptors and the F48E9 virus. The infected brain sections at 5 dpi were stained with MAA for SA $\alpha 2,3-$ Gal (upper panel, green), SNA for SA a2,6-Gal (lower panel, green), anti-NDV NP antibody (red) and DAPI (blue). Negative control staining was performed without the lectins. Arrows represent positive co-localized staining. Scale bars represent $50 \mu \mathrm{m}$. 
(a)

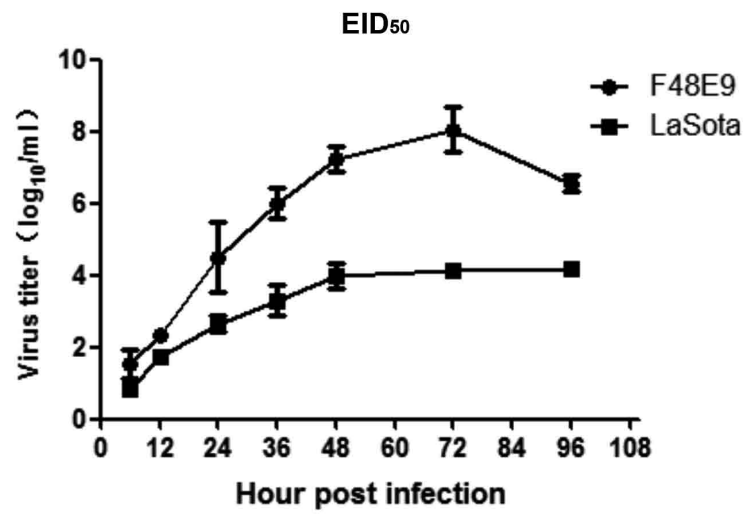

(b)

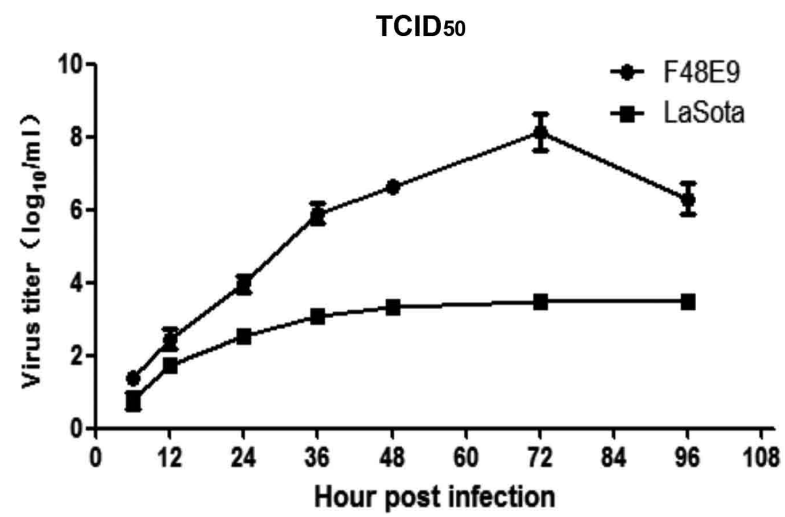

Fig. 3

Growth kinetics of different NDV strains in chicken primary neuronal cells

The cells were infected with strains F48E9 and LaSota at MOI of 0.01 and 1 , respectively. The supernatants were collected for viral titration at $12 \mathrm{~h}$ intervals. (a) The viral titers were tested in embryonated chicken eggs by an EID $_{50}$ assay. (b) The viral titers were tested in DF-1 cells by a TCID ${ }_{50}$ assay. The titers were determined according the method of Reed and Muench. The data are shown as the means \pm standard deviations (SD).

congestion (Fig. 1c left) as well as edema, neuronal necrosis, crowding of glial cells, vacuolization of neutrophils and astrocytosis (Fig. 1c right). However, these neuropathological changes were not observed in lentogenic LaSota- and PBS-mock-infected chicken brains (Fig. 1c). These results confirm that the velogenic F48E9 virus causes encephalitis in the chickens. CNS

Velogenic virus, but not lentogenic virus, replicates in the

To evaluate whether NDV was able to replicate in the CNS of infected chickens, 4-week-old SPF chickens were inoculated with velogenic or lentogenic viruses via intraocularnasal routes. The cerebrum, cerebellum and spinal cord tissue samples were collected at 1,3 and $5 \mathrm{dpi}$. The velogenic strain F48E9 was not detected in these three tissues at 1 dpi but was detected at 3 and 5 dpi. The F48E9 virus had the highest viral titer of $10^{5} \mathrm{TCID}_{50} / \mathrm{g}$ in the cerebrum and the lowest viral titer of $10^{3} \mathrm{TCID}_{50} / \mathrm{mg}$ in the spinal cord at $5 \mathrm{dpi}$ (Fig. 1d). The results indicate that the velogenic virus can efficiently replicate in the chicken CNS after a natural infection, and the cerebrum was more sensitive for viral replication than the cerebellum and the spinal cord. The lentogenic strain LaSota was not detectable in the cerebrum, cerebellum or spinal cord at any experimental day as assessed by either viral titration or qRT-PCR (data not shown). Moreover, the LaSota virus was also not found in the brains after the viral inoculation through an intracerebral route (data not shown). These data suggested that the lentogenic virus failed to replicate in the chicken CNS.
$N D V$ infection was related to the SA receptors in chicken brains

To determine whether the expression and distribution of SA molecules were related to the NDV infection, the SA a2,3Gal and SA $\alpha 2,6-$ Gal in chicken brain tissues were stained by lectin-SNA and MAA, respectively. Both SA receptors were detected in the brain tissues when the birds were infected with velogenic F48E9, lentogenic LaSota or the PBS control (Fig. 2a). Using mean optical density analysis, the hyperpallium showed the highest density in all groups. The SA distribution in the mesopallium, nidopallium and striatum regions was substantially similar but was observed to a lesser extent in the pyriform cortex (Fig. 2b). The densities of SA a2,6-Gal were not significantly different in the infected or the PBS control birds (Fig. 2c). The results indicated that the distribution of SA molecules was slightly different in the different regions of the chicken brains. Furthermore, in order to examine whether NDV infection related to the SA expression, a dual immunohistofluorescence assay was performed in the infected brain tissues. The granular patterns of SA $\alpha 2,3-\mathrm{Gal}$ and SA $\alpha 2,6-\mathrm{Gal}$ were co-localized with the viral NP protein in the F48E9-infected brain tissues at 5 dpi (Fig. 2d) but was not found in the LaSota-infected brains (data not shown). These data showed that NDV chickens brain infection may have a close relationship with both the SA 2,3-Gal and SA $\alpha 2,6-$ Gal receptors, at least in the velogenic virus infection. 
(a)

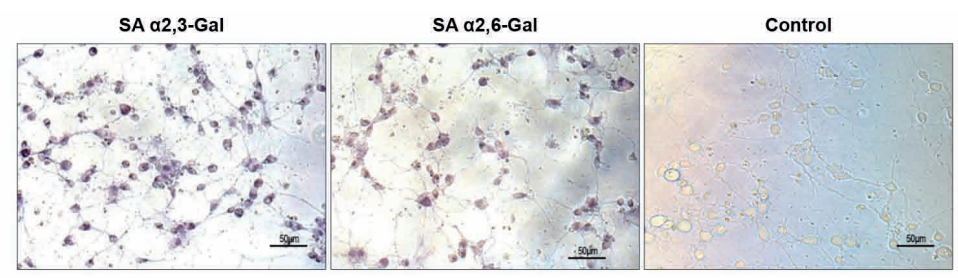

(b)
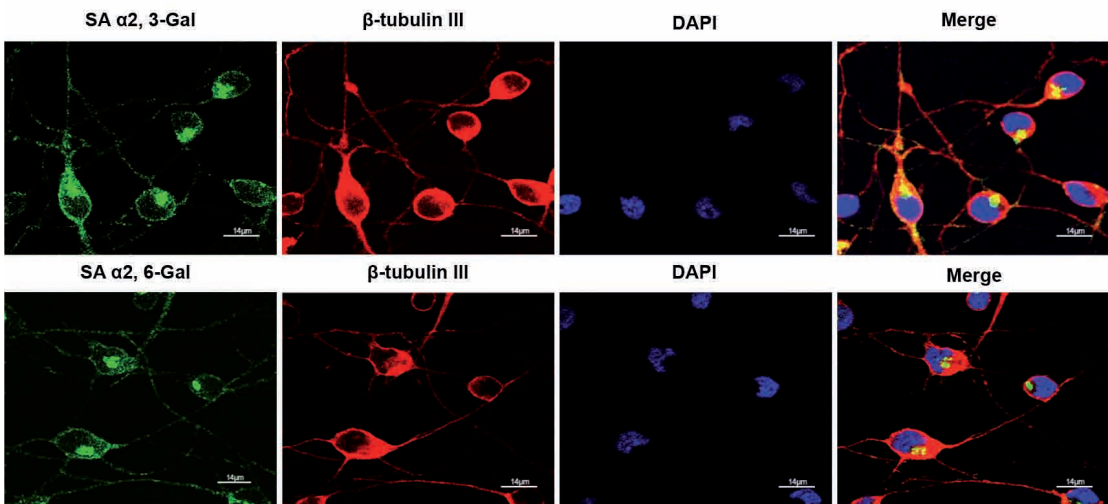

$\beta$-tubulin III

DAPI

Merge
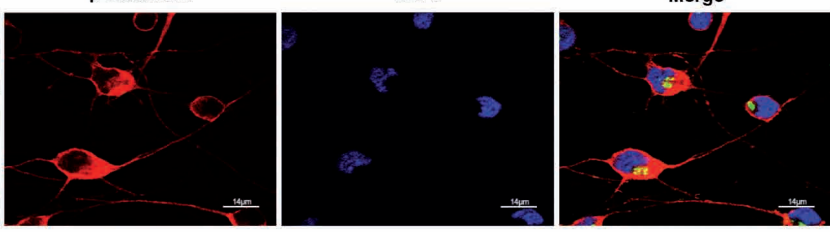

(c)
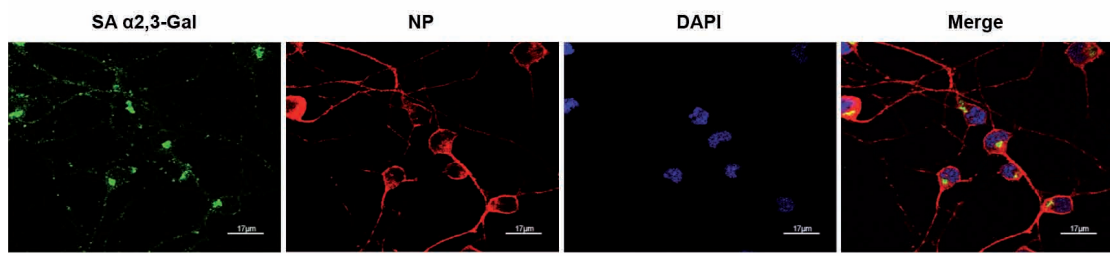

SA $\propto 2,6-G a$

NP

DAPI

Merge
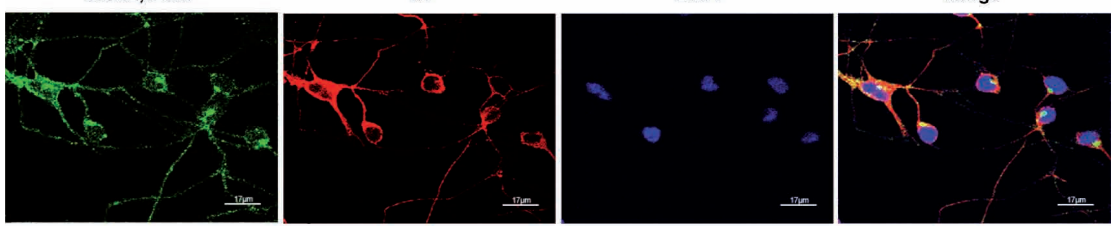

(d)
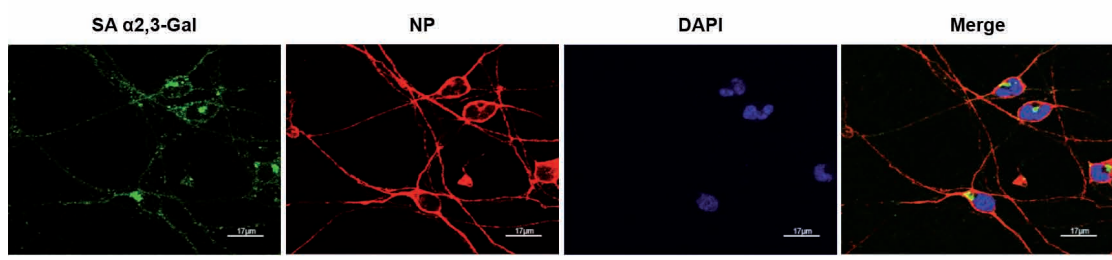

SA $\propto 2,6-$ Gal

NP

DAPI

Merge
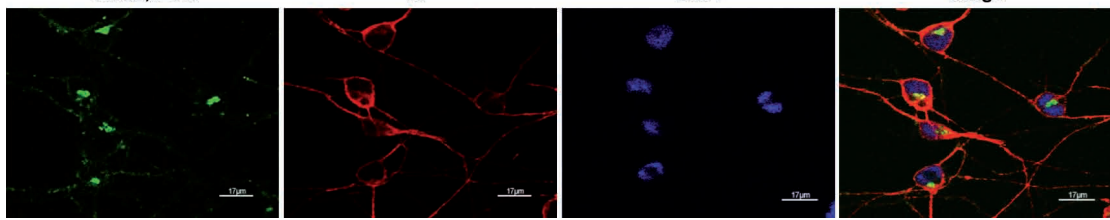

Fig. 4

Identification of SA receptors and NDV in chicken primary neuronal cells

(a) Chicken primary neuronal cells were prepared from 10-day-old embryos and cultured in 12-well plates with cover slips. The SA a2,3-Gal and SA a2,6-Gal receptors were determined by immunohistochemistry assay. Scale bars represent $50 \mu \mathrm{m}$. (b) Specificity of the primary neuronal cells and SA receptors. The cells were simultaneously stained with specific neuron marker $\beta$-tubulin III antibody (red), and MAA for SA a2,3-Gal (green) or SNA for SA a2,6-Gal (green) were observed by confocal microscopy. Scale bars represent $14 \mu \mathrm{m}$. (c) and (d) co-localization of SA a2,3-Gal and SA a2,6-Gal receptors with NDV. The cells infected with LaSota (c) or F48E9 (d) viruses were stained with an anti-viral NP antibody and treated with MAA or SNA. Samples were observed by confocal microscopy. The nuclei were counterstained with DAPI. Scale bars represent $17 \mu \mathrm{m}$. 
(a)

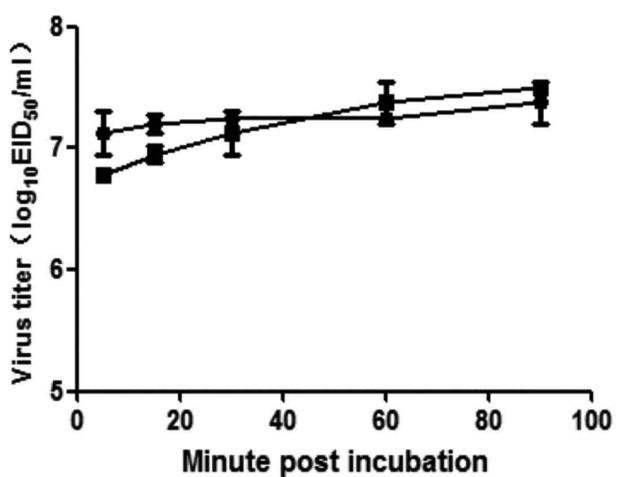

(b)

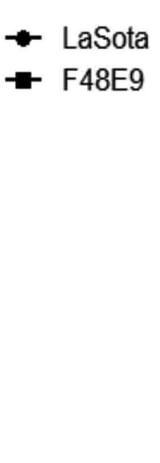

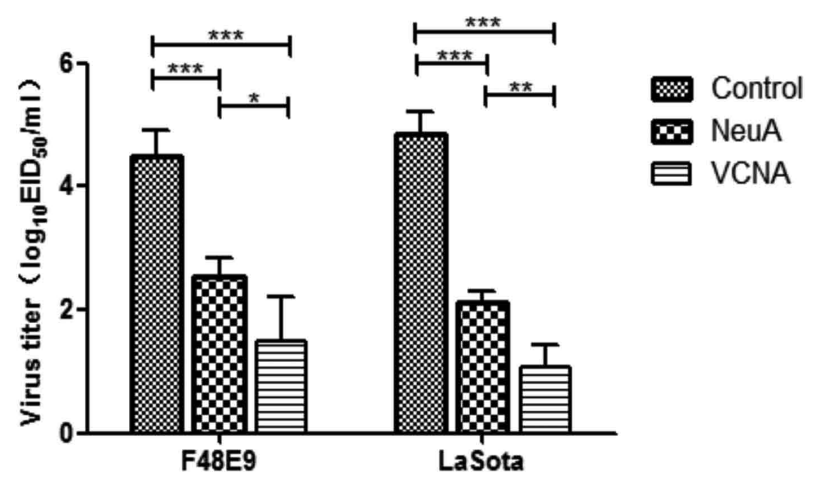

(c)

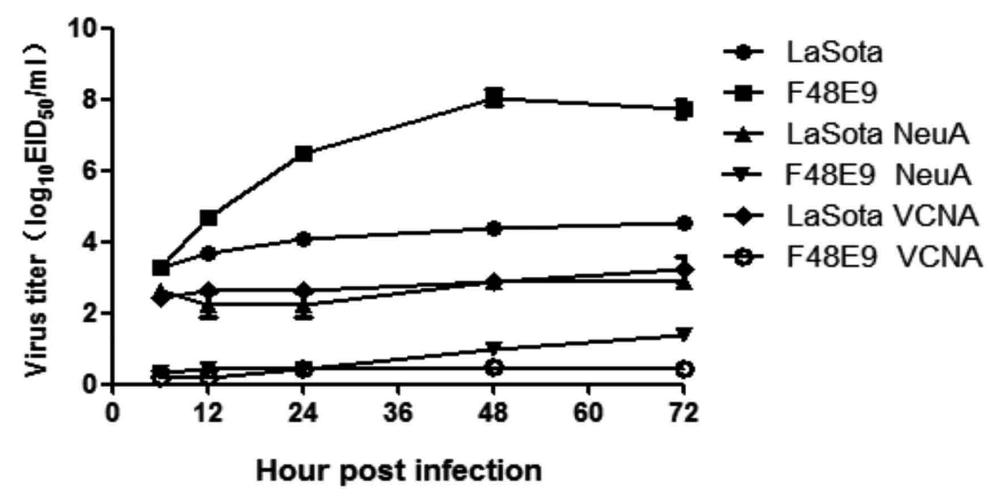

Fig. 5

Requirement for SA receptors for viral binding and replication in primary neuronal cells

(a) The capability of viral binding of the velogenic and lentogenic viruses. The cells were incubated with strains $\mathrm{F} 48 \mathrm{E} 9$ and LaSota at $\mathrm{MOI}$ of 50 at $4^{\circ} \mathrm{C}$. The cells were washed with PBS and harvested. The viruses were titrated by an $\mathrm{EID}_{50}$ assay. (b) SA receptors mediated viral binding activity. The cells were pretreated with sialidases at $37^{\circ} \mathrm{C}$ for $2 \mathrm{~h}$ and then were incubated with viruses at MOI of 50 at $4^{\circ} \mathrm{C}$ for $1 \mathrm{~h}$. The cells were collected for viral titration. (c) The viral replication was reduced by sialidase pretreatment. The cells were pretreated with sialidases and infected with viruses as described above in (b). The viruses in the cell supernatants were titrated. The differences in the viral titers were evaluated by a paired student's $t$ test, and significant differences are indicated by an asterisk $\left({ }^{*} \mathrm{P}<0.05,{ }^{* * *} \mathrm{P}<0.001\right)$. The data are shown as the means \pm standard deviations (SD).

\section{Replication of NDV in chicken primary neuronal cells}

We further examined viral replication in chicken primary neuronal cells. The cells were inoculated with strains F48E9 and LaSota. The viruses from the infected neuronal cells were titrated in embryonated chicken eggs and DF-1 cells at $12 \mathrm{~h}$ intervals. The viral titers of velogenic F48E9 in the neuronal cells gradually increased and reached the highest titers of $7.8 \log _{10} \mathrm{EID}_{50} / \mathrm{ml}$ (Fig. 3a) and $7.2 \log _{10} \mathrm{TCID}_{50} / \mathrm{ml}$ (Fig. 3b) at 72 hpi. In contrast, the titer of the lentogenic LaSota slightly increased in the neuronal cells (Fig. $3 \mathrm{a}$ and $3 b)$. These results indicate poor ability of lentogenic virus replication in the neuronal cells.

\section{SA expression in chicken primary neuronal cells infected} by NDV

To clarify whether NDV infection was related with SA receptors, expression and localization of SA and viral proteins in chicken primary neuronal cells were examined. The cells stained with lectin-SNA and MAA clearly showed strong expression of both SA 2,3-Gal or SA 2,6-Gal in immunocytochemistry assay (Fig. 4a). To confirm these data, the $\beta$-tubulin III, a neuron specific marker, was used to identify the neuronal cells by confocal immunofluorescence assay. The SA 2,3-Gal or SA 2,6-Gal receptors were expressed in dendrites and axons of the neurons (Fig. 4b). Next, we examined whether the expression of SA receptors was influenced by NDV infection. Both SA receptors were found to co-localize with viral protein-NP in the chicken 
primary neuronal cells. Expression of both, SA 2,3-Gal and SA 2,6-Gal, reduced after neuronal cells disruption in either lentogenic (Fig. 4c) or velogenic viruses (Fig. 4d).

\section{$S A$ receptors are important for viral binding and replica-} tion

First, in order to investigate the capability of NDV binding to neural cells, the chicken primary neuronal cells were incubated with the velogenic and lentogenic viruses at $4^{\circ} \mathrm{C}$ for 5, 15, 30, 60 and $90 \mathrm{~min}$. After the cells were washed with PBS, the cell-bound viruses were harvested and titrated by the EID $_{50}$ method. Both F48E9 and LaSota viruses were capable of binding to the neuronal cells, and there was no significant difference in the cell-bound viral titers between both viruses (Fig. 5a), indicating that the velogenic and lentogenic strains had similar abilities in cell binding.

Next, to verify whether the viral binding was mediated by SA receptors, SA $\alpha 2,3-\mathrm{Gal}$ and SA $\alpha 2,6-\mathrm{Gal}$ on the surface of chicken primary neuronal cells were eliminated by exogenous neuraminidases. As expected, there was a significant reduction in the number of cell-bound virus in all the treated groups compared with the corresponding control groups $(\mathrm{P}<0.05)$, and virus particles were more abundant on the surface of neuronal cells treated with NeuA than cells treated with VCNA (Fig. 5b).

Finally, we examined the viral replication in the neuronal cells pretreated with neuraminidases. After the viruses infected the chicken primary neuronal cells pretreated with the neuraminidases, the viruses in the cell culture supernatants were titrated by $\mathrm{EID}_{50}$. The replication results were generally consistent with the corresponding binding responses after treatment with the neuraminidases. The viral titers of F48E9 decreased significantly to approximately 50\% and $70 \%$ of the control after pretreatment with NeuA and VCNA, respectively. The same results could be observed for LaSota (Fig. 5c). The VCNA treatment for SA removal in the neuronal cells was more efficient than the NeuA treatment. This might be due to the neuraminidase activity of VCNA compared with that of NeuA. The results suggest that the SA a2,3-Gal and SA a2,6-Gal receptors were critical for NDVneuronal cell binding, however other SA molecules might be involved in this process.

\section{Discussion}

A viral receptor is a determinant of virus infection that affects host-range restriction and tropism (Pappas et al., 2010; Tumpey et al., 2007). Although sialylated molecules, such as gangliosides and glycoproteins, serve as NDV receptors (Ferreira et al., 2004), the role of SA a2,3-Gal and SA a2,6-Gal in the neurotropism of NDV is unknown. In this study, we showed that both $\alpha 2-3$ - and a2-6-linked SA were abundantly present in chicken brains and primary neuronal cells. Co-localization between both SA receptors and the velogenic virus were found in the infected chicken brain tissues. The replication and cell-binding capability of either velogenic or lentogenic strains were significantly reduced in neuraminidase-pretreated chicken primary neuronal cells. These data suggest that $\alpha 2-3$ - and $\alpha 2-6$-linked SA receptors are important for NDV neuroinfection.

SA $\alpha 2,3-G a l$ and SA $\alpha 2,6-G a l$ molecules are simultaneously present in chicken epithelial cells (Gambaryan et al., 2002) and susceptible tissues such as the respiratory tract, kidney and brain (Kuchipudi et al., 2009; Wang et al., 2012). Both receptors may allow effective infection of NDV to determine viral replication and virulence (Sánchez-Felipe et al., 2012; Villar and Barroso, 2006). This leads to the notion that SA may be closely related to the neuroinfection of NDV in the chicken brain. Avian influenza viruses have a strict binding preference for the SA a2,3-Gal receptor in birds. This preference may limit the replication of avian viruses in the respiratory tract of humans, in which SA a2,6-Gal predominates (Couceiro et al., 1993; Ito et al., 2000). Enzymatic removal of sialic acid residues from the surface of neuronal cells by treatment with sialidases, such as VCNA and NeuA, conferred a significant reduction in cell binding and replication of both the velogenic and lentogenic viruses. The cell binding and replication of NDV in the neuronal cells treated with VCNA were more efficient than that after treatment with NeuA. These data suggest that glycosphingolipids and O-glycoproteins might be involved in the viral entry process, as VCNA can perform a more extensive removal, including $\mathrm{N}$ - and $\mathrm{O}$-linked sialic acids. It is remarkable that viral binding and replication were not completely inhibited by both VCNA and NeuA. This raised a possibility of incomplete removal of the receptors in the neuronal cells after neuraminidase treatment. To examine sialidase activity, chicken RBCs were treated with VCNA and NeuA. No viral binding of NDV was observed (data not shown). This demonstrates that the neuraminidase substantially eliminated sialic acids from the RBCs. The incomplete inhibition of NDV binding by treatment with sialidases indicates that additional molecules may be involved in the viral binding to host cells (Guo et al., 1999). The entry of NDV into host cells is mediated by receptor-mediated endocytosis or direct fusion of the viral envelope with the plasma membrane to deliver the nucleocapsid into the cytoplasm (Cantin et al., 2007; San et al., 1999). Therefore, the requirement of a specific SA receptor in the initial interaction of NDV with host cells may be the priority.

Infection with the velogenic F48E9 virus but not the lentogenic LaSota virus caused neurological lesions in chicken brains during inoculation through the natural route. The F48E9 virus could be positively detected in the 
infected chicken brains by using immunohistochemistry and RT-PCR. However, the LaSota virus was undetectable. Moreover, using an intracerebral infection route in 1-dayold chicks, the LaSota virus was also not detected in the chicken brains (data not shown). These data indicate that replication of lentogenic virus was inefficient in the brain. This is consistent with a previous report (Kim et al., 2012). In contrast, both the F48E9 and LaSota viruses were able to replicate in chicken primary neuronal cells, even though the replication of the F48E9 virus was more efficient than that of the LaSota virus. The inability of lentogenic virus replication in the central nervous system may depend heavily on viral virulence, ability to cross the blood-brain barrier, reaching the cerebrospinal fluid, and efficiency of replication and spread in neurons and glial cells (Moura et al., 2016). Host defence factors such as the immune response may also be involved in the replication of lentogenic virus (Dortmans et al., 2011; Madansky and Bratt, 1981). The mechanism of replication of velogenic and lentogenic viruses in chicken CNS needs to be further investigated.

a2,3- and $\alpha 2,6$-linked sialic acids as NDV receptors were abundantly expressed in chicken brains and primary neuronal cells and were shown to be essential for the viral binding and replication of NDV. This is the first preliminary evidence that NDV uses SA $\propto 2,3-G a l$ and SA $\propto 2,6-$-Gal to infect neuronal cells in chickens. This should help to understand further NDV neurotropism in avian species.

Acknowledgments. This work was supported by the National Natural Science Foundation of China (31572533) and the Faculty Support Fund of the Northwest A \& F University (Z111021401).

\section{References}

Alexander DJ (2000): Newcastle disease and other avian paramyxoviruses. Rev. Sci. Tech. 19, 443-462. https://doi. org/10.20506/rst.19.2.1231

Alexander DJ, Senne DA (2008): Newcastle disease, other paramyxoviruses and pneumovirus infections. In Saif YM, Fadly AM, Glisson JR, McDougald LR, Nolan LK, Swayne DE (Eds): Diseases of Poultry. Iowa State University Press, Ames, IA, pp. 75-115.

Alexander DJ (1997): Newcastle disease and other avian Paramyxoviridae infection. In Calnek BQ (Eds): Diseases of Poultry. Iowa State University Press, Ames, IA, pp. 541-569.

Brown C, King DJ, Seal BS (1999): Pathogenesis of Newcastle disease in chickens experimentally infected with viruses of different virulence. Vet. Pathol. 36, 125-132. https://doi. org/10.1354/vp.36-2-125

Cantin C, Holguera J, Ferreira LE, Munoz-Barroso I (2007): Newcastle disease virus may enter cells by caveolae-mediated endocytosis. J. Gen. Virol. 88, 559-569. https://doi. org/10.1099/vir.0.82150-0
Cattoli G, Susta L, Terregino C, Brown C (2011): Newcastle disease: a review of field recognition and current methods of laboratory detection. J. Vet. Diagn. Invest. 23, 637-656. https://doi.org/10.1177/1040638711407887

Cerná A, Janega P, Martanovic P, Lisý M, Babál P (2002): Changes in sialic acid expression in the lung during intrauterine development of the human fetus. Acta Histochem. 104, 339-342. https://doi.org/10.1078/0065-1281-00669

Couceiro JNSS, Paulson JC, Baum LG (1993): Influenza virus strains selectively recognize sialyl oligosaccharides on human respiratory epithelium; the role of the host cell in selection of hemagglutin in receptor specificity. Virus Res. 29, 155-165. https://doi.org/10.1016/0168-1702(93) $90056-\mathrm{S}$

Dechecchi MC, Melotti P, Bonizzato A, Santacatterina M, Chilosi M, Cabrini G (2001): Heparan sulfate glycosaminoglycans are receptors sufficient to mediate the initial binding of adenovirus types 2 and 5. J. Virol. 75, 8772-8780. https:// doi.org/10.1128/JVI.75.18.8772-8780.2001

Delorme C, Brüssow H, Sidoti J, Roche N, Karlsson KA, Neeser JR, Teneberg S (2001): Glycosphingolipid binding specificities of rotavirus: identification of a sialic acid-binding epitope. J. Virol. 75, 2276-2287. https://doi.org/10.1128/ JVI.75.5.2276-2287.2001

Dortmans JC, Koch G, Rottier PJ, Peeters BP (2011): Virulence of Newcastle disease virus: what is known so far? Vet. Res. 42, 1-11. https://doi.org/10.1186/1297-9716-42-122

Dugan AS, Gasparovic ML, Atwood WJ (2008): Direct correlation between sialic acid binding and infection of cells by two human polyomaviruses (JC virus and BK virus). J. Virol. 82, 2560-2564. https://doi.org/10.1128/JVI.02123-07

Ecco R, Susta L, Afonso CL, Miller PJ, Brown C (2011): Neurological lesions in chickens experimentally infected with virulent Newcastle disease virus isolates. Avian Pathol. 40, 145152. https://doi.org/10.1080/03079457.2010.544289

Ferreira L, Villar E, Muñoz-Barroso I (2004): Gangliosides and $\mathrm{N}$-glycoproteins function as Newcastle disease virus receptors. Int. J. Biochem. Cell. Biol. 36, 2344-2356. https:// doi.org/10.1016/j.biocel.2004.05.011

Gambaryan A, Webster R, Matrosovich M (2002): Differences between influenza virus receptors on target cells of duck and chicken. Arch.Virol. 147, 1197-1208. https://doi. org/10.1007/s00705-002-0796-4

Guo C, Nakagomi O, Mochizuki M, Ishida H, Kiso M, Ohta Y, Suzuki T, Miyamoto D, Hidari RIPJ, Suzuki Y (1999): Ganglioside GMia on the cell surface is involved in the infection by human rotavirus KUN and MO strains. J. Biochem. 126, 683-688. https://doi.org/10.1093/oxfordjournals.jbchem.a022503

Ito T, Suzuki Y, Suzuki T, Takada A, Horimoto T, Wells K, Kida H, Otsuki K, Kiso M, Ishida H (2000): Recognition of $\mathrm{N}$-glycolylneuraminic acid linked to galactose by the a2,3 linkage is associated with intestinal replication of influenza a virus in ducks. J. Virol. 74, 9300-9305. https:// doi.org/10.1128/JVI.74.19.9300-9305.2000

Kim SH, Xiao S, Shive H, Collins PL, Samal SK (2012): Replication, neurotropism, and pathogenicity of avian paramyxovirus 
serotypes 1-9 in chickens and ducks. Plos One. 7, e34927. https://doi.org/10.1371/journal.pone.0034927

Kuchipudi SV, Nelli R, White GA, Bain M, Chang KC, Dunham S (2009): Differences in influenza virus receptors in chickens and ducks: Implications for interspecies transmission. J. Mol. Genet. Med. 3, 143-151. https://doi. org/10.4172/1747-0862.1000026

Lamb RA, Kolakofsky D (2007): Paramyxoviridae: the viruses and their replication. In Knipe DM, Howley PM, Griffin DE, Lamb RA, Martin MA, Roizman B, Straus SE (Eds): Fields Virology. Lippincott Williams \& Wilkins, Philadelphia, pp. 1449-1496.

Madansky CH, Bratt MA (1981): Noncytopathic mutants of Newcastle disease virus are defective in virus-specific RNA synthesis. J. Virol. 37, 317-327.

Moura VM, Susta L, Cardenasgarcia S, Stanton JB, Miller PJ, Afonso CL, Brown CC (2016): Neuropathogenic capacity of lentogenic, mesogenic, and velogenic Newcastle disease virus strains in day-old chickens. Vet. Pathol. 53, 53-64. https://doi.org/10.1177/0300985815600504

Neu U, Stehle T, Atwood WJ, Lambert PF (2009): The Polyomaviridae: contributions of virus structure to our understanding of virus receptors and infectious entry.Virology 384, 389-399. https://doi.org/10.1016/j.virol.2008.12.021

Pappas C, Viswanathan K, Chandrasekaran A, Raman R, Katz JM, Sasisekharan R, Tumpey TM (2010): Receptor specificity and transmission of $\mathrm{H} 2 \mathrm{~N} 2$ subtype viruses isolated from the pandemic of 1957. Plos One. 5, e11158. https://doi. org/10.1371/journal.pone.0011158

Reiner A, Perkel DJ, Mello CV, Jarvis ED (2004): Songbirds and the revised avian brain nomenclature. Ann. N. Y. Acad. Sci. 1016, 77-108. https://doi.org/10.1196/annals.1298.013

San Román KS, Villar E, Muñoz-Barroso I (1999): Acidic pH enhancement of the fusion of Newcastle disease virus with cultured cells. Virology 260, 329-341. https://doi. org/10.1006/viro.1999.9841

Sánchez-Felipe L, Villar E, Muñoz-Barroso I (2012): a2-3- and a2-6-N-linked sialic acids allow efficient interaction of Newcastle disease virus with target cells. Glycoconj. J. 29, 539-549. https://doi.org/10.1007/s10719-012-9431-0

Schnaar RL (1991): Glycosphingolipids in cell surface recognition. Glycobiology 1, 477-485. https://doi.org/10.1093/ glycob/1.5.477
Schultze B, Cavanagh D, Herrler G (1992): Neuraminidase treatment of avian infectious bronchitis coronavirus reveals a hemagglutinating activity that is dependent on sialic acid-containing receptors on erythrocytes. Virology 189, 792-794. https://doi.org/10.1016/0042-6822(92)90608-R

Stevens J, Blixt O, Tumpey TM, Taubenberger JK, Paulson JC, Wilson IA (2006): Structure and receptor specificity of the hemagglutinin from an $\mathrm{H} 5 \mathrm{~N} 1$ influenza virus. Science 312, 404-410. https://doi.org/10.1126/science.1124513

Suzuki Y (2005): Sialobiology of influenza: molecular mechanism of host range variation of influenza viruses. Biol. Pharm. Bull. 28, 399-408. https://doi.org/10.1248/bpb.28.399

Tumpey TM, Maines TR, Van HN, Glaser L, Solórzano A, Pappas C, Cox NJ, Swayne DE, Palese P, Katz JM (2007): A two-amino acid change in the hemagglutinin of the 1918 influenza virus abolishes transmission. Science 315, 655-659. https://doi.org/10.1126/science.1136212

Villar E, Barroso IM (2006): Role of sialic acid-containing molecules in paramyxovirus entry into the host cell: a mini review. Glycoconj. J. 23, 5-17. https://doi.org/10.1007/ $\underline{\text { s10719-006-5433-0 }}$

Viswanathan K, Chandrasekaran A, Srinivasan A, Raman R, Sasisekharan V, Sasisekharan R (2010): Glycans as receptors for influenza pathogenesis. Glycoconj. J. 27, 561-570. https://doi.org/10.1007/s10719-010-9303-4

Wang B, Liu B, Chen L, Zhang J, He H, Zhang H (2012): Qualitative and quantitative analyses of influenza virus receptors in trachea and lung tissues of humans, mice, chickens and ducks. Sci. China Life. Sci. 55, 612-617. https://doi. org/10.1007/s11427-012-4341-8

Wang X, Wang X, Jia Y, Wang C, Han Q, Lu ZH, Yang Z (2017): Adenoviral-expressed recombinant granulocyte monocyte colony-stimulating factor (GM-CSF) enhances protective immunity induced by inactivated Newcastle disease virus (NDV) vaccine. Antiviral. Res. 144, 322-329. https://doi. org/10.1016/j.antiviral.2017.07.004

Wu D, Huang W, Wang Y, Guan W, Li R, Yang Z, Zhong N (2014): Gene silencing of $\beta$-galactosamide $\alpha$-2,6-sialyltransferase 1 inhibits human influenza virus infection of airway epithelial cells. BMC Microbiol. 14, 78. https://doi. org/10.1186/1471-2180-14-78 